\title{
Estimation of health state utilities in breast cancer
}

\author{
Seon-Ha Kim ${ }^{\prime}$ \\ Min-Woo Jo² \\ Minsu Ock ${ }^{2}$ \\ Hyeon-Jeong Lee ${ }^{2}$ \\ Jong-Won Lee ${ }^{3,4}$ \\ 'Department of Nursing, College \\ of Nursing, Dankook University, \\ Cheonan, ${ }^{2}$ Department of Preventive \\ Medicine, University of Ulsan College \\ of Medicine, Seoul, ${ }^{3}$ Department of \\ Breast and Endocrine Surgery, Asan \\ Medical Center, Seoul, ${ }^{4}$ Department of \\ Surgery, University of Ulsan College \\ of Medicine, Seoul, South Korea
}

Correspondence: Min-Woo Jo Department of Preventive Medicine, University of Ulsan College of Medicine, 88, Olympic-ro 43-gil, Songpa-gu,

Seoul 05505, South Korea

Tel +82230104264

Fax +82 24772898

Email mdjominwoo@gmail.com
This article was published in the following Dove Press journal:

Patient Preference and Adherence

14 March 2017

Number of times this article has been viewed

Purpose: The aim of this study is to determine the utility of breast cancer health states using the standard gamble (SG) and visual analog scale (VAS) methods in the Korean general population. Materials and methods: Eight hypothetical breast cancer health states were developed based on patient education material and previous publications. Data from 509 individuals from the Korean general population were used to evaluate breast cancer health states using the VAS and the SG methods, which were obtained via computer-assisted personal interviews. Mean utility values were calculated for each human papillomavirus (HPV)-related health state.

Results: The rank of health states was identical between two valuation methods. SG values were higher than VAS values in all health states. The utility values derived from SG were 0.801 (noninvasive breast cancer with mastectomy and followed by reconstruction), 0.790 (noninvasive breast cancer with mastectomy only), 0.779 (noninvasive breast cancer with breast-conserving surgery and radiation therapy), 0.731 (invasive breast cancer with surgery, radiation therapy, and/or chemotherapy), 0.610 (locally advanced breast cancer with radical mastectomy with radiation therapy), 0.587 (inoperable locally advanced breast cancer), 0.496 (loco-regional recurrent breast cancer), and 0.352 (metastatic breast cancer).

Conclusion: Our findings might be useful for economic evaluation of breast cancer screening and interventions in general populations.

Keywords: breast neoplasm, Korea, quality-adjusted life years, quality of life

\section{Introduction}

Breast cancer is one of the most commonly diagnosed and the deadliest cancers among women worldwide, representing a quarter of all cancers diagnosed in women. ${ }^{1}$ Its incidence is highest in Europe and North America, although cases are on the rise in Africa and Asia. ${ }^{2,3}$ Over 200,000 new cancers are diagnosed per year. In 2013, breast cancer was the fifth most frequently diagnosed cancer in Korea, accounting for 7.7\% of all cancers diagnosed. ${ }^{4}$ Early detection and timely management of breast cancer are important as the social burden of breast cancer is substantial.

Decision makers are interested in both the efficacy and efficiency of early detection and treatment. Cost-utility analysis in economic evaluation was developed to compare the costs of a health care program and its beneficial impacts on both length and quality of life. ${ }^{5}$ Quality-adjusted life years are used as a utility measure and are calculated by multiplying the length of time spent in a particular health state by the utility weight associated with that health state. ${ }^{6}$

Quality weights (utility weights) in various breast cancer states are required to estimate quality-adjusted life years or evaluate the cost-utility of intervention for breast cancer. Two approaches have commonly been used to determine the quality weights: direct evaluation using the visual analog scale (VAS), standard gamble (SG), and time trade-off (TTO) techniques or indirect approaches using multi-attribute health state classification systems (eg, EQ-5D [EuroQol five dimensions questionnaire]) and quality weight tariffs. ${ }^{7}$ 
Previous utility studies have evaluated a range of health states (screening related, adverse events, treatment, and metastatic breast cancer), population groups (professional, patient, and general population), and valuation methodologies. ${ }^{8-11}$ According to the study of Peasgood et al ${ }^{9}$ reviewing 49 articles, SG was the most frequently used with $59.9 \%$, followed by VAS (22.2\%), TTO (11.1\%), and EQ-5D $(34.5 \%)$ in the estimation of utility of metastatic breast cancer health states, and VAS was the most frequently used with $34.5 \%$, followed by SG (28\%), EQ-5D (16.9\%), and TTO $(15.3 \%)$ in the estimation of utility of early breast cancer health states. Health utilities in breast cancer states have been shown to vary significantly depending on valuation method, study population, health states, and location. ${ }^{8,9}$ Policy decision makers need to be aware of country-specific utility weight when evaluating health care interventions.

Therefore, the purpose of this study was to determine the utility values of breast cancer health states using the SG and VAS methods in the Korean general population. We anticipate that our findings will have utility in improving cost-utility analysis in Korea. This study was performed as part of a larger research project for the economic evaluation of cancer screening programs.

\section{Materials and methods}

\section{Health states}

A draft of the breast cancer health state scenarios used in this study was created by two investigators ( $\mathrm{MO}$ and S-HK) based on guidance for breast cancer patients, ${ }^{12}$ the 5 th Korean guideline for the management of breast cancer, ${ }^{13}$ and a breast cancer white paper from the Korean breast cancer society. ${ }^{14}$ One breast specialist from a tertiary teaching hospital (J-WL) reviewed and modified the draft authored by two other medical doctors (M-WJ and MO).

Each breast cancer health state was designed to reflect common treatment regimens considering breast cancer staging. A total of eight hypothetical scenarios were made: 1) noninvasive breast cancer with mastectomy only; 2) noninvasive breast cancer with mastectomy and followed by reconstruction; 3 ) noninvasive breast cancer with breastconserving surgery and radiation therapy; 4 ) invasive breast cancer with surgery (mastectomy or breast-conserving surgery), radiation therapy, and/or chemotherapy; 5) locally advanced breast cancer with radical mastectomy with radiation therapy; 6) inoperable, locally advanced breast cancer; 7) loco-regional recurrent breast cancer; and 8) metastatic breast cancer. Each scenario consisted of four parts: diagnosis, symptoms, treatment, and progression and prognosis. The progress and prognosis section included treatment side effects, required follow-up visits, 5-year survival probability, and recurrence probability. All scenarios for breast cancer health states are described in detail in Supplementary materials.

\section{Study participants}

The target population consisted of adults aged $\geq 19$ years living in Korea (except Jeju Island). A total of 509 representative individuals were randomly selected from this target population using a multistage stratified quota method. A sample quota was assigned to each of the 15 regions according to population structure (number of population in region, sex, age, and level of education), as defined in the June 2013 resident registration data available through the Ministry of Administration and Security, South Korea.

\section{Interviews}

Data were collected using computer-assisted, face-to-face interviews. Interviewers were employed by a research agency and were experienced in several valuation studies. In total, 54 interviewers performed all interviews. Interviewers were trained in how to apply the VAS and SG methods using laptops and practiced in pairs before conducting the field survey. Surveys were performed from March to April in 2016.

Participants were initially asked about their region, sex, age, and educational level. Next, participants evaluated eight scenarios and a dead state on the VAS scale. Eight scenarios were displayed randomly regardless of severity, and the dead state was the last scenario to be rated on the VAS scale. Then, each participant was randomly assigned one of eight scenarios evaluated using the SG approach. After the two rounds of evaluation, participants were asked other background questions regarding income, ambulatory care visits within the preceding 2 weeks, admissions during the preceding 12 months, and current diseases. Visual aids were used to help respondents understand questions, and larger sizes of health state descriptions were additionally provided for participants with visual impairments. This study was approved by the Institutional Review Board of Asan Medical Center (approval no: S2016-0015) with exemption for written informed consent. To minimize the collection of personal information in a minimum risk study, we applied for exemption from written informed consent, and the study proceeded with oral informed consent from each participant for this study.

\section{Valuation methods}

We used VAS and SG as valuation methods. In the VAS approach, respondents were asked to rate health states on 
a scale from 0 to 100 , with 0 representing the worst imaginable health and 100 indicating the best imaginable health. In the $\mathrm{SG}$ valuation tasks, respondents distinguished between health states that were "better than death" states and "worse than death" states. For states considered worse than death, an SG evaluation of the scenario was completed. For states considered better than death, interviewers attempted to determine the respondent's point of indifference between a certain outcome of the target health state, "i", over an average life expectancy and receiving treatment with the uncertain prospect of two possible outcomes: either the subject is returned to full health and lives for an average life expectancy (probability " $P$ ”) or the subject dies immediately (probability " $1-P$ "). ${ }^{15}$ The chances of the best outcome were initially evaluated at $50 \%$ and increased or decreased by $10 \%$ according to the subject's response. If respondents changed their preference, the probability of the best outcome was increased or decreased by $5 \%$ according to the subject's response (eg, $50 \% \rightarrow 60 \% \rightarrow 70 \% \rightarrow$ [if preference changed] $65 \%$ ). The minimum probability interval was $5 \%$.

\section{Data analysis}

Descriptive statistics were performed for sociodemographic factors. Utility values according to VAS for health states were given by the formula: $(x-d) /(100-d)$, where $x$ corresponds to the VAS values of the health state and $d$ corresponds to the VAS values of death. ${ }^{15}$ For states better than death, health state values were given as the probability of normal health at the respondent's point of indifference according to the SG method. For states worse than death, utility values for all health states were censored at zero.

Mean utility values according to demographic factors and clinical information were compared using Student's $t$-test and analysis of variance. All statistical analyses were conducted using SAS version 9.2 software (SAS Institute, Cary, NC, USA). $P$-values $<0.05$ were considered statistically significant.

\section{Results}

A total of 509 respondents (mean age, 45.7 [standard deviation: 14.1 ] years, $48.9 \%$ male) were successfully interviewed. Of these, $9 \%$ had current comorbidities. Clinical and demographic characteristics of the respondents are shown in Table 1.

Utility values for breast cancer scenarios are shown in Table 2. The rank of scenarios was identical between the two valuation methods. Scenario 2 (noninvasive breast cancer with mastectomy and followed by reconstruction) was assigned the highest utility values at 0.681 using VAS and at
Table I Characteristics of participants $(n=509)$

\begin{tabular}{lll}
\hline Characteristics & $\mathbf{n}$ & $\%$ \\
\hline Sex & & \\
Male & 249 & 48.9 \\
Female & 260 & 51.1 \\
Age group (years) & & \\
I9-29 & 91 & 17.9 \\
$30-39$ & 91 & 17.9 \\
$40-49$ & 110 & 21.6 \\
$50-59$ & 101 & 19.8 \\
$\geq 60$ & 116 & 22.8 \\
Education level & & \\
Middle school or below & 44 & 8.6 \\
High school & 239 & 47.0 \\
College or above & 226 & 44.4 \\
Monthly income (million Korean won) & & \\
$<3$ & 105 & 20.6 \\
$3-5$ & 253 & 49.7 \\
$>5.0$ & 151 & 29.7 \\
Ambulatory care visit in the past 2 weeks & & \\
Yes & 46 & 9.0 \\
No & 463 & 91.0 \\
Hospitalization in the past I2 months & & \\
Yes & 11 & 2.2 \\
No & 498 & 97.8 \\
Morbidity & & \\
Yes & 46 & 91.0 \\
No & 463 & \\
\hline
\end{tabular}

0.804 using SG, while scenario 8 (metastatic breast cancer) was assigned the lowest utility values at 0.170 using VAS and at 0.352 using SG. The mean utility weight calculated according to the $\mathrm{SG}$ was greater than that calculated from the VAS for all health states. Difference in utility values between the two valuation methods ranged from 0.117 (noninvasive breast cancer with breast-conserving surgery and radiation therapy) to 0.182 (metastatic breast cancer). Severe health states tended to have larger differences between the two approaches.

Utility values derived from SG were 0.801 (noninvasive breast cancer with mastectomy and followed by reconstruction), 0.790 (noninvasive breast cancer with mastectomy only), 0.779 (noninvasive breast cancer with breast-conserving surgery and radiation therapy), 0.731 (invasive breast cancer with surgery, radiation therapy, and/or chemotherapy), 0.610 (locally advanced breast cancer with radical mastectomy with radiation therapy), 0.587 (inoperable locally advanced breast cancer), 0.496 (loco-regional recurrent breast cancer), and 0.352 (metastatic breast cancer).

Comparisons of utility weights according to demographic and clinical information are shown in Table 3 . The mean utility weights calculated from the SG did not differ significantly with sex, age, educational level, and monthly income. However, 
Table 2 Utility values of breast cancer scenarios derived from the VAS and SG approaches

\begin{tabular}{|c|c|c|c|c|c|c|c|}
\hline \multirow[t]{2}{*}{ Health states } & \multirow[t]{2}{*}{$\mathbf{n}$} & \multicolumn{3}{|l|}{ VAS } & \multicolumn{3}{|l|}{ SG } \\
\hline & & Mean & SD & Median & Mean & SD & Median \\
\hline I. Noninvasive breast cancer with mastectomy only (AJCC 7th 0 ) & 509 & 0.669 & 0.199 & 0.694 & 0.790 & 0.265 & 0.900 \\
\hline $\begin{array}{l}\text { 2. Noninvasive breast cancer with mastectomy and followed by } \\
\text { reconstruction (AJCC 7th 0) }\end{array}$ & 509 & 0.681 & 0.199 & 0.700 & 0.804 & 0.260 & 0.900 \\
\hline $\begin{array}{l}\text { 3. Noninvasive breast cancer with breast-conserving surgery and radiation } \\
\text { therapy }(\mathrm{AJCC} \text { 7th } 0)\end{array}$ & 509 & 0.662 & 0.201 & 0.684 & 0.779 & 0.261 & 0.900 \\
\hline $\begin{array}{l}\text { 4. Invasive breast cancer with surgery (mastectomy or breast-conserving } \\
\text { surgery), radiation therapy, and/or chemotherapy (AJCC 7th I, II) }\end{array}$ & 509 & 0.579 & 0.202 & 0.600 & 0.731 & 0.255 & 0.800 \\
\hline $\begin{array}{l}\text { 5. Locally advanced breast cancer with radical mastectomy and radiation } \\
\text { therapy (AJCC 7th IIIA, IIIB) }\end{array}$ & 509 & 0.435 & 0.178 & 0.451 & 0.610 & 0.261 & 0.650 \\
\hline 6. Inoperable locally advanced breast cancer (AJCC 7th IIIC) & 509 & 0.415 & 0.173 & 0.438 & 0.587 & 0.259 & 0.650 \\
\hline 7. Loco-regional recurrent breast cancer & 509 & 0.333 & 0.176 & 0.357 & 0.496 & 0.260 & 0.500 \\
\hline 8. Metastatic breast cancer (AJCC 7th IV) & 509 & 0.170 & 0.220 & 0.167 & 0.352 & 0.275 & 0.300 \\
\hline
\end{tabular}

Abbreviations: VAS, visual analog scale; SG, standard gamble; SD, standard deviation; AJCC, American Joint Committee on Cancer.

respondents who had visited ambulatory care in the preceding 2 weeks tended to have higher scores than those who had not visited ambulatory care. Respondents with current comorbidities rated all health states, except metastatic breast cancer, significantly higher than those without disease.

\section{Discussion}

In this study, quality weights for eight breast cancer health states using SG and VAS were elicited from 509 respondents of the general population in South Korea. We evaluated the preferences of a relatively large sample and various scenarios using direct valuation methods. The range of breast cancer quality weight values was from 0.352 (metastatic breast cancer) to 0.804 (noninvasive breast cancer with mastectomy and followed by reconstruction), with more severe states consistently found to have lower values.

Our scenarios were based on both medical guidelines ${ }^{13}$ and patient education material. ${ }^{12}$ When evaluating the

Table 3 Utility weights of breast cancer scenarios derived from the SG approach according to sociodemographic factors

\begin{tabular}{|c|c|c|c|c|c|c|c|c|c|c|c|c|c|c|c|c|}
\hline \multirow[t]{2}{*}{ Variables } & \multicolumn{2}{|c|}{ Scenario I } & \multicolumn{2}{|c|}{ Scenario 2} & \multicolumn{2}{|c|}{ Scenario 3} & \multicolumn{2}{|c|}{ Scenario 4} & \multicolumn{2}{|c|}{ Scenario 5} & \multicolumn{2}{|c|}{ Scenario 6} & \multicolumn{2}{|c|}{ Scenario 7} & \multicolumn{2}{|c|}{ Scenario 8} \\
\hline & Mean & SD & Mean & SD & Mean & SD & Mean & SD & Mean & SD & Mean & SD & Mean & SD & Mean & SD \\
\hline \multicolumn{17}{|l|}{ Sex } \\
\hline Male & 0.786 & 0.265 & 0.804 & 0.259 & 0.778 & 0.265 & 0.735 & 0.250 & 0.618 & 0.258 & 0.579 & 0.259 & 0.502 & 0.258 & 0.357 & 0.277 \\
\hline Female & 0.795 & 0.266 & 0.803 & 0.261 & 0.780 & 0.258 & 0.728 & 0.260 & 0.603 & 0.264 & 0.594 & 0.259 & 0.490 & 0.262 & 0.347 & 0.273 \\
\hline \multicolumn{17}{|l|}{ Age group (years) } \\
\hline $19-29$ & 0.816 & 0.244 & 0.824 & 0.247 & 0.796 & 0.253 & 0.742 & 0.239 & 0.599 & 0.243 & 0.570 & 0.240 & 0.465 & 0.249 & 0.325 & 0.259 \\
\hline $30-39$ & 0.746 & 0.303 & 0.770 & 0.291 & 0.728 & 0.300 & $0.7 \mid 4$ & 0.272 & 0.626 & 0.260 & 0.582 & 0.244 & 0.488 & 0.228 & 0.360 & 0.250 \\
\hline $40-49$ & 0.797 & 0.267 & 0.792 & 0.283 & 0.787 & 0.260 & 0.719 & 0.269 & 0.597 & 0.277 & 0.556 & 0.291 & 0.483 & 0.280 & 0.340 & 0.278 \\
\hline $50-59$ & 0.820 & 0.217 & 0.824 & 0.213 & 0.800 & 0.224 & 0.747 & 0.239 & 0.614 & 0.247 & 0.608 & 0.239 & 0.509 & 0.252 & 0.374 & 0.290 \\
\hline$\geq 60$ & 0.773 & 0.284 & 0.807 & 0.258 & 0.781 & 0.265 & 0.735 & 0.257 & 0.616 & 0.275 & 0.615 & 0.269 & 0.525 & 0.279 & 0.359 & 0.292 \\
\hline \multicolumn{17}{|l|}{ Education level } \\
\hline High school or below & 0.790 & 0.269 & 0.799 & $0.26 I$ & 0.780 & 0.260 & 0.734 & 0.254 & 0.598 & 0.264 & 0.594 & 0.259 & 0.500 & 0.265 & $0.34 I$ & 0.278 \\
\hline College or above & 0.791 & 0.259 & 0.809 & 0.259 & 0.778 & 0.263 & 0.728 & 0.257 & 0.625 & 0.257 & 0.577 & 0.259 & 0.490 & 0.255 & 0.365 & 0.271 \\
\hline \multicolumn{17}{|c|}{ Monthly income (million Korean won) } \\
\hline$<3$ & 0.806 & 0.263 & 0.820 & 0.251 & 0.806 & 0.255 & 0.756 & 0.256 & 0.631 & 0.264 & 0.603 & 0.269 & 0.522 & 0.283 & 0.358 & 0.281 \\
\hline $3-5$ & 0.778 & 0.268 & 0.791 & 0.267 & 0.765 & 0.263 & 0.713 & 0.251 & 0.590 & 0.254 & 0.571 & 0.248 & 0.477 & 0.243 & 0.339 & 0.268 \\
\hline$>5.0$ & 0.800 & 0.264 & 0.814 & 0.254 & 0.785 & 0.262 & 0.745 & 0.260 & 0.629 & 0.269 & 0.602 & 0.270 & 0.509 & 0.270 & 0.370 & 0.283 \\
\hline \multicolumn{17}{|c|}{ Ambulatory care visit in the past 2 weeks } \\
\hline Yes & 0.828 & 0.237 & 0.851 & 0.220 & 0.837 & 0.232 & 0.798 & 0.224 & $0.685^{*}$ & 0.287 & $0.668^{*}$ & 0.304 & $0.614 * *$ & 0.268 & 0.417 & 0.293 \\
\hline No & 0.786 & 0.268 & 0.799 & 0.263 & 0.773 & 0.263 & 0.725 & 0.257 & $0.603 *$ & 0.257 & $0.579 *$ & 0.253 & $0.484 * *$ & 0.257 & 0.345 & 0.273 \\
\hline \multicolumn{17}{|c|}{ Hospitalization in the past 12 months } \\
\hline Yes & 0.750 & 0.259 & 0.791 & 0.202 & 0.759 & 0.238 & 0.695 & 0.258 & 0.655 & 0.260 & $0.64 I$ & 0.253 & 0.573 & 0.263 & 0.418 & 0.320 \\
\hline No & 0.791 & 0.266 & 0.804 & 0.261 & 0.780 & 0.262 & 0.732 & 0.255 & 0.609 & $0.26 I$ & 0.586 & 0.259 & 0.494 & 0.260 & 0.350 & 0.274 \\
\hline \multicolumn{17}{|l|}{ Morbidity } \\
\hline Yes & $0.865^{*}$ & 0.199 & $0.874 * *$ & 0.175 & $0.855^{* *}$ & 0.192 & 0.830 ** & 0.173 & $0.695^{*}$ & 0.255 & 0.691 ** & 0.237 & $0.590 * *$ & 0.256 & 0.348 & 0.256 \\
\hline No & $0.783^{*}$ & 0.270 & $0.797^{* *}$ & 0.266 & $0.772 * *$ & 0.266 & $0.721^{\text {** }}$ & 0.260 & $0.602 *$ & 0.260 & $0.576^{* *}$ & 0.259 & $0.486 * *$ & 0.259 & 0.352 & 0.277 \\
\hline
\end{tabular}

Notes: $* P$-value $<0.05 ; * * P$-value $<0.01$.

Abbreviations: SG, standard gamble; SD, standard deviation. 
preferences of the general population, these scenarios need to be explained in a manner than can be universally understood. ${ }^{16}$ Thus, the use of patient education material enabled respondents to better understand the different breast cancer health states. We included information related to prognosis, including the probability of survival and recurrence in addition to symptoms and treatments in each scenario, as prognosis may influence the utility weights of different cancer states. ${ }^{17,18}$

In the present survey, males were also recruited to evaluate hypothetical breast cancer states. Previous studies, especially surveys of breast cancer patients, asked females to value breast cancer states ${ }^{8}$ as breast cancer is considered a female disease. However, though rare, breast cancer is observed in males. ${ }^{19}$ From a societal perspective, a preference toward general public opinion, including both female and male, on a health state is recommended by economic evaluation guidelines..$^{20}$ Therefore, in this study, males were asked to evaluate breast health state imaging of his own or relative's state. Interestingly, no statistically significant different scenarios in VAS and SG values were observed according to sex, as reported by a previous study. ${ }^{16}$

We used VAS and SG approaches to determine the utility weights of the general population for different breast cancer health states. The VAS approach is simple and easy to administer and has demonstrated utility in evaluating health states and obtaining ordinal preferences. However, as cardinal preferences obtained using the VAS are prone to biases, the VAS should never be used alone. ${ }^{21}$ The SG is a classic method for measuring respondent cardinal utilities under conditions of uncertainty and is based directly on the fundamental axioms of the utility theory. ${ }^{22}$ Subjects from the general population are unable to easily understand the concept of probability, and several studies have reported the SG method to be as feasible and acceptable as TTO in eliciting social health preferences. ${ }^{23-25}$ In order to ensure familiarity with health states, respondents first rated health states using the VAS method, with visual props used in the SG valuation tasks.

As previous breast cancer utility studies ${ }^{8,9}$ have used different methodologies, scenarios, and subject groups, direct comparisons are not possible. In this study, SG utility scores were higher than VAS values, corroborating previous research reports. ${ }^{22,26}$ Peasgood et $\mathrm{al}^{9}$ also reported significantly higher VAS values than those obtained by the SG method in early breast cancer and metastatic breast cancer models. In the Schleinitz general population study using SG, the mean utility values in stages I-IV without estrogen receptor and IV with estrogen receptor were $0.68,0.61,0.56,0.42$, and 0.41 , respectively. ${ }^{17}$ Cappelli et $\mathrm{al}^{27}$ compared women's preferences for breast cancer treatment between breast cancer patients, high-risk relatives, and general population. The mean utility values of lumpectomy and radiation were $0.78,0.73$, and 0.68 in cancer patients, high-risk relatives, and general population, respectively, similar to utility of our scenario 3 (mean $=0.779$ ). ${ }^{27}$ In the study by Shih et al ${ }^{28}$ of oncology nurses, median utility values ranged from 0.299 to 0.371 in distant recurrence health states and from 0.336 to 0.376 in loco-regional recurrence health states.

A previous breast cancer study using the indirectEQ-5D-3L (EuroQol five dimensions questionnaire-three level) method conducted in Korean breast cancer patients reported utility values varying from 0.925 (American Joint Committee on Cancer [AJCC] stage 0) to 0.895 (AJCC stage 3 ). ${ }^{29}$ The utility differences between the findings of this study and previous studies in Korea ${ }^{29}$ may be attributable to the adaptation of patient and a ceiling effect of EQ-5D-3L. Respondents with current comorbidities in this study reported higher values in most scenarios than those without comorbidities. Patients have previously shown to report higher values for health states than individuals selected from the community. ${ }^{9,30}$ As several studies have reported a ceiling effect of EQ-5D-3L, ${ }^{31-33}$ EQ-5D-3L may lack sensitivity in specific conditions.

There are several limitations of this study. 1) We purposely reduced the number of scenarios to minimize the cognitive burden on respondents. Consequently, as some scenarios (eg, scenario 5) simultaneously included a variety of situations, it may be difficult for respondents to make fully informed decisions. 2) We did not collect data regarding the total number of persons asked to complete the survey; we were unable to determine the response rate or compare characteristics between responders and nonresponders. However, the general characteristics of the respondents was similar to the general population in Korea due to the multistage stratified quota sampling method used. 3) We did not apply SG method "for states considered worse than death" to reduce burden of subjects and interviewers. In all scenarios except scenario $8(9.23 \%),<2 \%$ of states indicated that their status was "less than death". The utility weights in breast cancer states in this study may be overestimated.

\section{Conclusion}

Our findings indicate the feasibility of evaluating a range of breast cancer state descriptions using either the VAS or the SG methods in the Korean general population. The VAS and SG methods may have utility in the economic evaluation of breast cancer screening and interventions for the general population and breast cancer patients. 


\section{Acknowledgment}

This work was supported by a grant from the National R\&D Program for Cancer Control, Ministry of Health and Welfare, Republic of Korea (number of study: 1520140).

\section{Author contributions}

All authors contributed toward data analysis, drafting and critically revising the paper and agree to be accountable for all aspects of the work.

\section{Disclosure}

The authors report no conflicts of interest in this work.

\section{References}

1. Ferlay J, Soerjomataram I, Dikshit R, et al. Cancer incidence and mortality worldwide: sources, methods and major patterns in GLOBOCAN 2012. Int J Cancer. 2015;136(5):E359-E386.

2. DeSantis CE, Bray F, Ferlay J, Lortet-Tieulent J, Anderson BO, Jemal A. International variation in female breast cancer incidence and mortality rates. Cancer Epidemiol Biomarkers Prev. 2015;24(10):1495-1506.

3. Servick K. Breast cancer. Breast cancer: a world of differences. Science. 2014;343(6178):1452-1453.

4. Korea Central Cancer Registry, National Cancer Center [webpage on the Internet]. Annual report of cancer statistics in Korea in 2013, Ministry of Health and Welfare, 2015. Available from: http://ncc.re.kr/main. ncc?uri=english/sub04_Statistics. Accessed December 19, 2016. Korean.

5. Dolan P, Gudex C, Kind P, Williams A. Valuing health states: a comparison of methods. J Health Econ. 1996;15(2):209-231.

6. Whitehead SJ, Ali S. Health outcomes in economic evaluation: the QALY and utilities. Br Med Bull. 2010;96:5-21.

7. Gold MR, Siegel JE, Russell LB, Weinstein MC. Cost-Effectiveness in Health and Medicine. Oxford: Oxford University Press; 1996.

8. Hao Y, Wolfram V, Cook J. A structured review of health utility measures and elicitation in advanced/metastatic breast cancer. Clinicoecon Outcomes Res. 2016;8:293-303.

9. Peasgood T, Ward SE, Brazier J. Health-state utility values in breast cancer. Expert Rev Pharmacoecon Outcomes Res. 2010;10(5):553-566.

10. Chie WC, Huang CS, Chen JH, Chang KJ. Utility assessment for different clinical phases of breast cancer in Taiwan. J Formos Med Assoc. 2000;99(9):677-683.

11. Milne RJ, Heaton-Brown KH, Hansen P, Thomas D, Harvey V, Cubitt A. Quality-of-life valuations of advanced breast cancer by New Zealand women. Pharmacoeconomics. 2006;24(3):281-292.

12. Ahn SE. Guidance for Breast Cancer Patient. 5th ed. Seoul: Youngchang Publication; 2012. Korean.

13. Korean Breast Cancer Society \& Korean Cancer Study Group [homepage on the Internet]. 5th Korean guideline for the management of breast cancer. Available from: http://www.kbcs.or.kr/. Accessed December 19, 2016. Korean.

14. Korean Breast Cancer Society. Breast Cancer White Paper. Seoul: Korean Breast Cancer Society; 2013. Available from: http://www.kbcs. or.kr/sub02/sub04.html\#none. Accessed December 19, 2016. Korean.

Patient Preference and Adherence

\section{Publish your work in this journal}

Patient Preference and Adherence is an international, peer-reviewed, open access journal that focuses on the growing importance of patient preference and adherence throughout the therapeutic continuum. Patient satisfaction, acceptability, quality of life, compliance, persistence and their role in developing new therapeutic modalities and compounds to optimize
15. Torrance GW. Measurement of health state utilities for economic appraisal. J Health Econ. 1986;5(1):1-30.

16. Ock M, Park JY, Son WS, Lee HJ, Kim SH, Jo MW. Estimation of utility weights for human papilloma virus-related health states according to disease severity. Health Qual Life Outcomes. 2016;14(1):163.

17. Schleinitz MD, DePalo D, Blume J, Stein M. Can differences in breast cancer utilities explain disparities in breast cancer care? J Gen Intern Med. 2006;21(12):1253-1260.

18. Kennedy ED, Detsky AS, Llewellyn-Thomas HA, et al. Can the standard gamble be used to determine utilities for uncertain health states? An example using postoperative maintenance therapy in Crohn's disease. Med Decis Making. 2000;20(1):72-78.

19. Kreiter E, Richardson A, Potter J, Yasui Y. Breast cancer: trends in international incidence in men and women. Br J Cancer. 2014;110(7): 1891-1897.

20. Claxton K, Walker S, Palmer S, et al. Appropriate perspectives for health care decisions. CHE Research Paper 54. York: Centre for Health Economics, University of York; 2010.

21. Torrance GW, Feeny D, Furlong W. Visual analog scales: do they have a role in the measurement of preferences for health states? Med Decis Making. 2001;21(4):329-334.

22. Drummond MF, Sculpher MJ, Torrance GW, O'Brien BJ, Stoddart GL. Methods for the Economic Evaluation of Health Care Programmes. Oxford; New York, NY: Oxford University Press; 2005.

23. Wee HL, Li SC, Xie F, et al. Validity, feasibility and acceptability of time trade-off and standard gamble assessments in health valuation studies: a study in a multiethnic Asian population in Singapore. Value Health. 2008;11(suppl 1):S3-S10.

24. Jo MW, Kwon DS, Kim SH, Kil SR, Lee S. Validity and reliability of Korean EQ-5D valuation study using a computer-assisted standard gamble method. Korean J Health Promot. 2010;10:105-112. Korean.

25. Kim SH. The Utility Estimation for EQ-5D-5L Health States Using Standard Gamble Method [dissertation]. Ulsan: University of Ulsan; 2012.

26. Morimoto T, Fukui T. Utilities measured by rating scale, time trade-off, and standard gamble: review and reference for health care professionals. J Epidemiol. 2002;12(2):160-178.

27. Cappelli M, Surh L, Humphreys L, et al. Measuring women's preferences for breast cancer treatments and BRCA1/BRCA2 testing. Qual Life Res. 2001;10(7):595-607.

28. Shih V, Chan A, Xie F, Ko Y. Health state utility assessment for breast cancer. Value Health Reg Issues. 2012;1(1):93-97.

29. Kim SH, Jo MW, Lee JW, Lee HJ, Kim JK. Validity and reliability of EQ-5D-3L for breast cancer patients in Korea. Health Qual Life Outcomes. 2015;13:203.

30. Dolan P, Olsen JA, Menzel P, Richardson J. An inquiry into the different perspectives that can be used when eliciting preferences in health. Health Econ. 2003;12(7):545-551.

31. Johnson JA, Pickard AS. Comparison of the EQ-5D and SF-12 health surveys in a general population survey in Alberta, Canada. Med Care. 2000;38(1):115-121.

32. Kim SH, Kim HJ, Lee SI, Jo MW. Comparing the psychometric properties of the EQ-5D-3L and EQ-5D-5L in cancer patients in Korea. Qual Life Res. 2012;21(6):1065-1073.

33. Kim TH, Jo MW, Lee SI, Kim SH, Chung SM. Psychometric properties of the EQ-5D-5L in the general population of South Korea. Qual Life Res. 2013;22(8):2245-2253.

\section{Dovepress}

clinical outcomes for existing disease states are major areas of interest for the journal. This journal has been accepted for indexing on PubMed Central. The manuscript management system is completely online and includes a very quick and fair peer-review system, which is all easy to use. Visit http://www. dovepress.com/testimonials.php to read real quotes from published authors. 